\title{
Stimulated-emission pumping enabling sub-diffraction-limited spatial resolution in coherent anti-Stokes Raman scattering microscopy
}

\author{
Carsten Cleff, ${ }^{*}$ Petra Groß ${ }^{\dagger}$ and Carsten Fallnich \\ Institute of Applied Physics, Westfälische Wilhelms-Universität Münster, Corrensstraße 2, 48149 Münster, Germany
}

Herman L. Offerhaus and Jennifer L. Herek

Optical Sciences Group, MESA + Research Institute for Nanotechnology, University of Twente, P.O. Box 217, Enschede NL-7500 AE, The Netherlands

\author{
Kai Kruse, Willem P. Beeker, Chris J. Lee, ${ }^{\ddagger}$ and Klaus-Jochen Boller \\ Laser Physics and Nonlinear Optics Group, MESA+ Research Institute for Nanotechnology, University of Twente, \\ P.O. Box 217, Enschede NL-7500 AE, The Netherlands \\ (Received 8 January 2013; published 25 March 2013)
}

\begin{abstract}
We present a theoretical investigation of stimulated emission pumping to achieve sub-diffraction-limited spatial resolution in coherent anti-Stokes Raman scattering (CARS) microscopy. A pair of control light fields is used to prepopulate the Raman state involved in the CARS process prior to the CARS signal generation. Efficient prepopulation is achieved by employing a path via an electronic or vibrational state. Thereby, the buildup of a coherence between the ground and Raman state during irradiation by the light fields for CARS signal generation is prevented, resulting in the suppression of the CARS intensity by more than $99.8 \%$. Two-dimensional spatial excitation profiles have been calculated using donut-shaped spatial profiles for the control light fields, thus, an intensity-dependent narrowing of the CARS excitation profiles below the diffraction limit. Using computergenerated test images we demonstrate a resolution beyond the diffraction limit for CARS microscopy, which is scalable by the control light field intensity, similar to stimulated emission depletion microscopy.
\end{abstract}

DOI: 10.1103/PhysRevA.87.033830

PACS number(s): 42.30.-d, 78.20.Bh, 78.47.jh, 87.64.-t

\section{INTRODUCTION}

Nonlinear microscopy techniques are developing rapidly as they allow the acquisition of three-dimensional images without the need for staining. Purely parametric nonlinear microscopy techniques such as second [1] and third harmonic [2] generation microscopy already offer excellent images. But, nonlinear vibrational microscopy techniques, such as coherent anti-Stokes Raman scattering (CARS) microscopy [3,4], offer additional benefits, i.e., chemical selective imaging due to the interaction with vibrational states of the sample under test. Applications of CARS microscopy are widely spread, covering, e.g., the monitoring of physiological processes in biological systems [5], drug dissolution in pharmaceutical tablets [6], and the investigation of semiconductor and metal nanostructures [7].

However, for many samples, interesting processes take place at scales smaller than the diffraction limit. An investigation of such processes using far-field optical microscopy can only be performed with fluorescence-based techniques, as, for the time being, these are the only techniques that allow a spatial resolution well below the diffraction limit; prominent examples being stimulated emission depletion microscopy (STED) [8], photoactivated localization microscopy (PALM) [9], and stochastic optical reconstruction microscopy (STORM) [10].

\footnotetext{
*c.clef01@uni-muenster.de

†Present address: Institute for Physics, Carl von Ossietzky Universitat, 26111 Oldenburg, Germany.

${ }^{\ddagger}$ Present address: FOM Institute DIFFER, Edisonbaan 14, Nieuwegein NL-3439 MN, The Netherlands.
}

Especially with STED microscopy, video rate imaging with sub-diffraction-limited spatial resolution was demonstrated, which enabled the visualization of intracellular physiological processes, such as synaptic vesicle movement inside the axons of neurons [11]. However, these attempts require staining of the sample, which may alter the sample dynamics or may even be toxic for biological samples [12]. In nonbiological samples, such as semiconductor nanostructures, staining often is not possible or leads to significant alteration of the sample properties. Autofluorescence in such materials is not often possible, or based on defects, meaning that structures with low defect rates are hardly visible with fluorescence microscopy. Therefore, sub-diffraction-limited spatial resolution is desired for nonlinear microscopy techniques that do not require staining or autofluorescence.

On the topic of sub-diffraction-limited spatial resolution in far-field CARS microscopy, different approaches have been presented using focal volume engineering [13,14], structured illumination [15], incoherent coupling of vibrational states [16], and intensity-dependent frequency shifts due to Rabi oscillations [17]. While the first method is the one to be most easily realized in experiments, it can only enhance the resolution by a factor of about 2. Similar restrictions apply to structured illumination, which, in addition, requires an ambitious wide-field CARS microscope [18]. The last two of the above-mentioned schemes provide a resolution enhancement which directly depends on the intensity of a control light field, allowing, in principle, unlimited resolution improvement, which is in practice limited by noise, acquisition time, and sample damage. On the other hand, specific sample properties are required for these schemes to function, such 
as strong incoherent coupling between vibrational states, or a long coherence lifetime, allowing for a coherent population transfer resulting in Rabi oscillations. For a more general approach, we developed a scheme based on ground state depletion [19] that allows for a saturable suppression of the CARS intensity with common sample properties. In that scheme the CARS intensity suppression is limited to $75 \%$ leaving a diffraction-limited background in a CARS image. We have shown that this can be removed by generating a difference image between two CARS images, one with and one without an applied control light field, leaving only the sub-diffraction-limited information, but the noise sensitivity is enhanced during this postprocessing.

Here, we present a scheme allowing the CARS signal to be suppressed by more than $99.8 \%$. This scheme may provide sub-diffraction-limited spatial CARS microscopy with no need for postprocessing of the CARS images. In contrast to some of our previous works [16,17] this approach is based solely on the effectively incoherent optical stimulation of population transfer. In principle this scheme is applicable to any sample that is suited for CARS microscopy, as no sample specific properties, such as incoherent nonradiative transitions, or coherent optical population transfer is required. This approach is based on stimulated emission pumping [20,21], i.e., a pair of pulsed control light fields are used to introduce a population transfer via a higher vibrational or electronic state from the ground state to the Raman state that is probed by the CARS process. The resulting equalization of the ground state and Raman state populations leads to a complete suppression of the resonant CARS intensity in a saturable process. This allows to improve the spatial resolution beyond the diffraction limit, while the image quality is rather robust against noise compared to the ground state depletion scheme [19]. The CARS suppression and resulting spatial resolution enhancement is similar to the STED process known from fluorescence microscopy, but, in contrast to STED, it does not require any staining of the sample.

\section{THEORETICAL FRAMEWORK}

CARS electric field generation is calculated numerically using a density matrix formalism describing a four-level energy system (see Fig. 1). The level system represents a ground state $|1\rangle$, a vibrational state $|2\rangle$ involved in the CARS process (which we refer to as the Raman state), an electronic state $|3\rangle$, and a second vibrational state $|4\rangle$. While the electronic state is needed to provide the virtual states (dotted lines in Fig. 1) for the CARS process, it also acts as a control state to suppress CARS signal generation. The vibrational state $|4\rangle$ is required for the CARS process but it is included to offer an alternative control state to the electronic state. Such vibrational states are typically found in close vicinity to Raman states [22] and, therefore, the level scheme used describes the general properties of a molecular system.

Optical transitions between the ground state and the vibrational state $|1\rangle-|4\rangle$, and the electronic state $|1\rangle-|3\rangle$, as well as the transition between the Raman state and the vibrational state $|2\rangle-|4\rangle$, and the electronic state $|2\rangle-|3\rangle$ are dipole allowed. All other transitions are dipole forbidden. The resulting CARS intensity is calculated from the off-diagonal

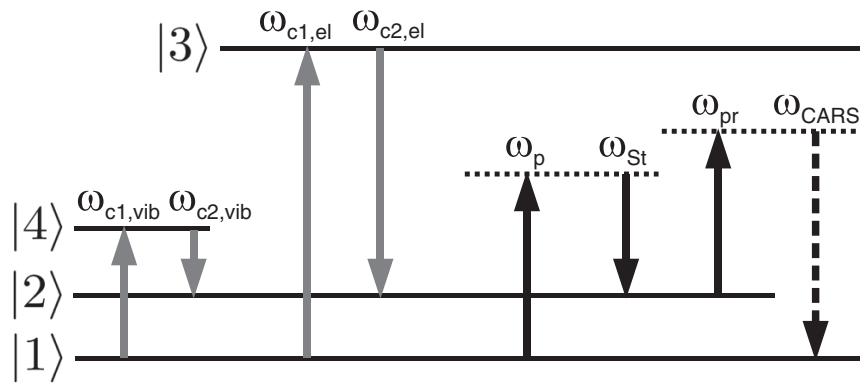

FIG. 1. Energy diagram of the CARS process and involved states: $|1\rangle$ is the ground state which is initially fully occupied. State $|2\rangle$ is a vibrational state involved in the CARS process which we refer to as the Raman state. $|3\rangle$ is the upper electronic state acting as a control state. An additional control state is added by incorporating a second vibrational state $|4\rangle$. The population distribution is manipulated by a pair of control light fields $\left(\omega_{c 1, \mathrm{el}}+\omega_{c 2, \text { el }}\right.$ or $\left.\omega_{c 1, \text { vib }}+\omega_{c 2, \text { vib }}\right)$ transferring population from the ground state to the Raman state $|2\rangle$ and thus preventing CARS intensity generation $\left(\omega_{\text {CARS }}\right)$ by the pump $\left(\omega_{p}\right)$, Stokes $\left(\omega_{\mathrm{St}}\right)$, and probe $\left(\omega_{\mathrm{pr}}\right)$ light fields.

elements of the density matrix [23]. A detailed description of the corresponding differential equations for the populations and coherences as well as their evaluation to calculate the CARS intensity are given by Beeker et al. [16]. The arrows in Fig. 1 indicate the irradiated and emitted light fields: For CARS, three pulsed light fields — which we refer to as pump, Stokes, and probe light fields-with frequencies $\omega_{p}, \omega_{\mathrm{St}}$, and $\omega_{\mathrm{pr}}$, respectively, are irradiated onto the four-level system. The pump and Stokes light fields are chosen to be two-photon resonant with the transition from the ground state to the Raman state $|1\rangle-|2\rangle$ in order to achieve a population transfer from $|1\rangle$ to $|2\rangle$, as well as the buildup of a coherence $\rho_{21}$ between those states. The interaction between the coherence $\rho_{21}$ and the probe light field generates the CARS electric field at the anti-Stokes frequency $\omega_{\mathrm{CARS}}=\omega_{p}-\omega_{\mathrm{St}}+\omega_{\mathrm{pr}}$. Although the four-level energy system referred to describes a molecular system, it is, in principle, applicable to any CARS sample, ranging from organic and inorganic molecules to quantum dots and nanomaterials.

In order to reduce the CARS intensity in a STED-like approach to achieve a sub-diffraction-limited excitation volume, the sample is prepared prior to the CARS process by applying a pair of pulsed control light fields (with frequencies $\omega_{c 1}$ and $\left.\omega_{c 2}\right)$ in addition to the pump, Stokes, and probe light fields. These control light fields are also two-photon resonant with the $|1\rangle-|2\rangle$ transition, such that, again, population transfer from the ground state to the Raman state is achieved. By adjusting the light field frequencies $\omega_{c 1}$ and $\omega_{c 2}$ such that the population transfer is either via the electronic level $|3\rangle$ or via the vibrational level $|4\rangle$, population is transferred to $|2\rangle$ with high efficiency. The population transfer is effectively incoherent, such that the population is equally distributed between the ground state $|1\rangle$, the control state $|3\rangle$ or $|4\rangle$, and the Raman state $|2\rangle$. Optically induced population transfer is typically found to be effectively incoherent under experimental conditions similar to those assumed in our calculations, as the pulse durations of the exciting pulses are typically longer than 
the coherence lifetimes. Nevertheless, since the stimulation of the population transfer is driven by a coherent light source, some coherence between states might be generated, while the population transfer still resembles the properties of an incoherent population transfer, e.g., equal population of the involved states. Using a delay between the control light fields and the pump, Stokes, and probe light fields we ensure that any coherence $\rho_{21}$ that might be generated by the control light fields will have decayed by the time of CARS generation. Thus, when the light fields for CARS generation arrive, the ground state $|1\rangle$ and Raman state $|2\rangle$ are equally populated and no coherence $\rho_{21}$ is present. As an equal population of the ground state $|1\rangle$ and Raman state $|2\rangle$ inhibits a coherence buildup and population transfer between these states by the pump and Stokes light fields, CARS signal generation is suppressed.

For the numerical calculation of the system dynamics, a Runge-Kutta algorithm of fourth order with fixed time steps of $0.5 \mathrm{fs}$ is used and the calculation was performed for a time interval of $100 \mathrm{ps}$.

The transition frequencies are chosen as follows: the $|1\rangle-|2\rangle$ two-photon resonant transition is set to $47 \mathrm{THz}\left(1568 \mathrm{~cm}^{-1}\right.$, $6379 \mathrm{~nm})$, which is within the regime of typical Raman transitions $\left(0-3400 \mathrm{~cm}^{-1}\right.$ [22]). The transition from the ground state to the vibrational state $|1\rangle-|4\rangle$ is set to $97 \mathrm{THz}\left(3236 \mathrm{~cm}^{-1}\right.$, $3091 \mathrm{~nm})$. As transitions from the ground state to an electronic state are typically found in the UV, the $|1\rangle-|3\rangle$ transition is set to $1000 \mathrm{THz}\left(33333 \mathrm{~cm}^{-1}, 300 \mathrm{~nm}\right)$. The coherence lifetime is chosen to be $5 \mathrm{ps}$ for the states $|2\rangle$ and $|4\rangle$ and $1 \mathrm{ps}$ for the electronic state $|3\rangle$. The lifetimes of the states $|2\rangle,|3\rangle$, and $|4\rangle$ are chosen to be $1 \mathrm{~ns}$.

To allow for a clear temporal separation of the effect of the control light field pair and the CARS excitation light fields, high population and coherence lifetimes are assumed for this work. Generally, the population and coherence lifetimes of CARS samples are found in a range from 10 to 1000 ps [24-28] and from 100 fs to 10 ps [29,30], respectively. However, this does not restrict the applicability of the presented scheme. In general, it can be said that as long as the delay between the control pulse pair and the CARS excitation pulses is short enough to ensure that the ground state $|1\rangle$ and the Raman state $|2\rangle$ are equally populated at the time of the CARS process, the CARS emission can be suppressed in a saturable way. We will show that a certain minimum delay is beneficial to avoid an interaction between a residual coherence $\rho_{21}$ generated by the control pulses and the probe pulse, resulting in an additional CARS signal, though a minimum delay is not required to achieve a saturable suppression of a CARS signal. This means that CARS signal suppression can also be provided by this scheme, if no delay between the control pulse pair and the pulses for CARS excitation is used. Consequently, CARS signal suppression can also be achieved for CARS samples with much shorter population lifetimes, e.g., around 10 ps. In addition, the applicability of the scheme to samples with shorter population and coherence lifetimes is validated using different sets of parameters for the prototype four-level system (population lifetimes varying between 10 and $1000 \mathrm{ps,}$ coherence lifetimes varying between $100 \mathrm{fs}$ and 5 ps). In all cases qualitatively similar results are found, i.e., the control light field pair leads to a strong suppression of CARS signal generation.
The pulse durations of the pump, Stokes, and probe light fields are $2 \mathrm{ps}$ (half width at $1 / e^{2}$ amplitude), while the duration of the control light fields is chosen to be $10 \mathrm{ps}$. The frequencies of the CARS excitation fields are set to $\omega_{p}=335 \mathrm{THz}$ $(895 \mathrm{~nm}), \omega_{\mathrm{St}}=288 \mathrm{THz}(1040 \mathrm{~nm})$, and $\omega_{\mathrm{pr}}=395 \mathrm{THz}$ (759 nm) ensuring two-photon resonance of the pump and Stokes light fields with the $|1\rangle-|2\rangle$ transition. We investigate two different frequency sets for the control light field pair: First we use the electronic state $|3\rangle$ as the control state and choose one control light field $\left(\omega_{c 1, \mathrm{el}}\right)$ to be resonant with the $|1\rangle-|3\rangle$ transition $\left(\omega_{c 1, \mathrm{el}}=1000 \mathrm{THz}, 300 \mathrm{~nm}\right)$ and the other $\left(\omega_{c 2, \mathrm{el}}\right)$ to be resonant with the $|3\rangle-|2\rangle$ transition $\left(\omega_{c 2, \mathrm{el}}=957 \mathrm{THz}\right.$, $313 \mathrm{~nm})$. In the second case we use the vibrational state $|4\rangle$ as the control state and thus the control light fields are chosen to be resonant with the $|1\rangle-|4\rangle\left(\omega_{c 1 \text {,vib }}=97 \mathrm{THz}, 3091 \mathrm{~nm}\right)$ and $|4\rangle-|2\rangle\left(\omega_{c 2, \text { vib }}=50 \mathrm{THz}, 5996 \mathrm{~nm}\right)$ transitions, respectively. In order to achieve a prepopulation of the Raman state the control pulse pair is irradiated $30 \mathrm{ps}$ prior to the pump, Stokes, and probe light fields. This value of the delay is chosen to allow a clear separation between the effects of the control fields and the light fields for CARS excitation.

A general discussion of the results only requires knowledge of the population distribution of the four-level system and the relative intensity values. However, to give an estimation of the intensity values needed for an experimental verification, the intensity values used were rescaled into $\mathrm{W} / \mathrm{cm}^{2}$ based on an exemplary sample [31]. This exemplary sample is based on quantum dots, which provide a high transition probability between states in combination with a high damage threshold. In addition to the control intensities used, the corresponding pulse energy fluence is specified, as the main limiting factor of the achievable spatial resolution, i.e., damage of the sample, consists of two contributions: damage through heating, which is related to the pulse energy fluence irradiated on the sample, and multiphoton damage and ionization, which is related to the applied peak intensity. All transition probabilities of the four-level energy system are set to the same value to provide a comparability between the two sets of control light field pairs. However, for population transfer from the ground state to a vibrational state in biological samples often a much lower transition probability is found [32-34], resulting in the requirement of higher control light field intensities of up to several orders of magnitude. As a result of the increased intensity requirements, the application of the presented schemes in biological samples might be limited as sample damage might occur before a significant resolution enhancement would be realized. However, for the investigation of inorganic molecules or nanomaterials, which category of sample is used here to estimate the required control intensities, the damage thresholds and transition probabilities are typically sufficiently high, such that sub-diffraction-limited spatial resolution should be experimentally realizable. In the case of samples that do not allow a direct implementation of this scheme because the required intensity values would result in sample damage, inorganic materials could be used as markers to provide sub-diffraction-limited spatial resolution. Though this would compromise the benefit of marker-free CARS microscopy, the use of quantum dots or other inorganic markers might be less toxic to biological samples than fluorescence dyes, such that samples could be investigated with 

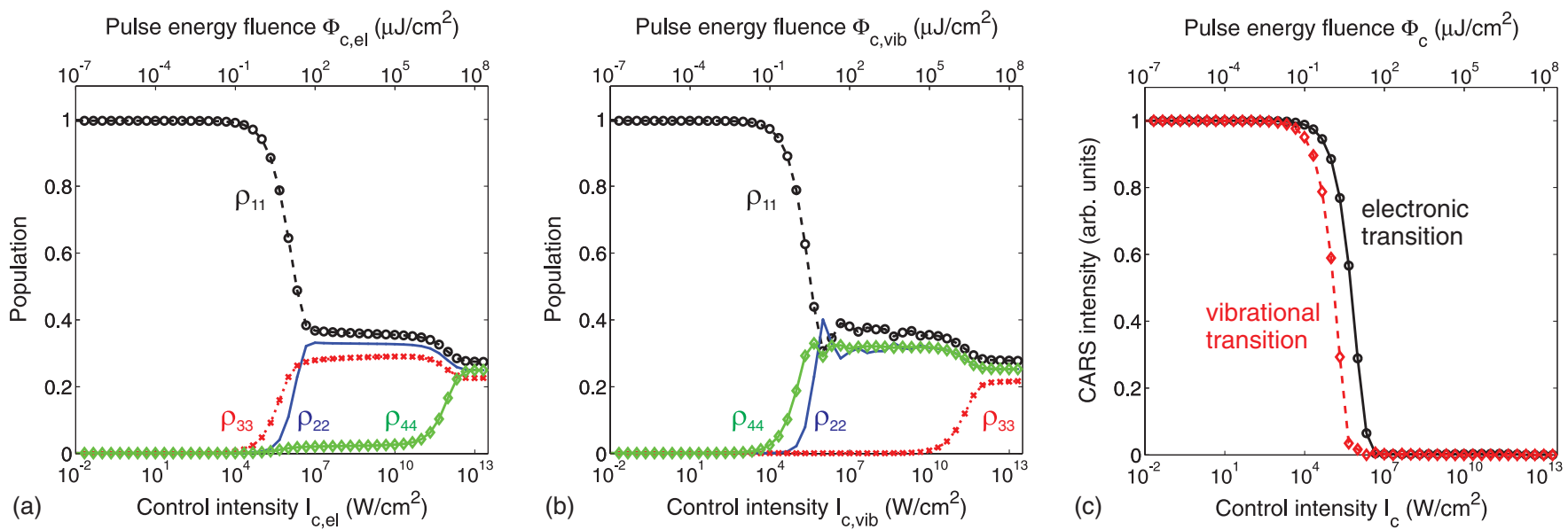

FIG. 2. (Color online) Population distribution of the states as a function of the intensity of the control light fields for (a) the electronic state $|3\rangle$ and (b) the vibrational state $|4\rangle$ acting as the control state. (c) Resulting CARS intensity as function of the intensity of the control light fields for the case in which the vibrational state acts as the control state (red dashed curve) or in which the electronic state acts as the control state (black solid curve).

sub-diffraction-limited spatial resolution that are not accessible with STED microscopy.

\section{RESULTS}

\section{A. Suppression of CARS intensity}

Figures 2(a) and 2(b) show the diagonal density matrix elements $\rho_{11}, \rho_{22}, \rho_{33}$, and $\rho_{44}$ at the end of each calculation (after $100 \mathrm{ps}$ ), representing the final population densities of the states, as a function of the control light field intensities in cases that the electronic state or the vibrational state is used as the control state, respectively. In both cases, increasing the control light field intensity $I_{c}=I_{c 1}+I_{c 2}=2 I_{c 1}$ leads to a strong population transfer from the ground state $|1\rangle$ to the Raman state $|2\rangle$ via the control state. When the electronic state acts as the control state [Fig. 2(a)], the population of state $|2\rangle$ is achieved via the electronic state $|3\rangle$, and once an equal population of the three involved states (ground state $|1\rangle$, electronic level $|3\rangle$, and Raman state $|2\rangle$ ) is reached, they remain equalized even when the control light field intensity is further increased. In the case in which the vibrational state $|4\rangle$ acts as the control state [Fig. 2(b)], the population of each of the three involved states (ground state $|1\rangle$, vibrational level $|4\rangle$, and Raman state $|2\rangle$ ) shows a damped oscillation as a function of the control light intensity. This oscillation arises because the coherence lifetime between the vibrational state $|4\rangle$ and ground state $|1\rangle$ is chosen to be five times longer than that between the electronic state $|3\rangle$ and the ground state $|1\rangle$, which leads to a barely noticeable partially coherent population transfer (strongly damped Rabi oscillation). The oscillatory effect vanishes if the pulse duration of the control light fields is increased. In both cases, at very high control light field intensities, off-resonant population transfer occurs which populates the last unpopulated state as well $(|4\rangle$ or $|3\rangle$, respectively).

The resulting CARS intensity as a function of the control light field intensity is shown in Fig. 2(c) for the case in which the vibrational state $|4\rangle$ is used as the control state (dashed red curve), and for the case in which the electronic state $|3\rangle$ acts as the control state (solid black curve). In both cases the CARS intensity is suppressed strongly when the populations of ground state, Raman state, and control state reach equalization, and when further increasing the control intensity, the CARS intensity suppression saturates at more than $99.8 \%$. Compared to the case of the electronic state $|3\rangle$ acting as the control state (solid black curve), in the case of the vibrational state $|4\rangle$ acting as the control state (dashed red curve), the CARS intensity is depleted by $50 \%$ at approximately five times lower control intensities. This results from the longer coherence lifetime of the vibrational state $|4\rangle$. The longer coherence lifetime also leads, via the oscillating population difference $\rho_{11}-\rho_{22}$, to a CARS intensity suppression showing oscillations when the populations of the ground and Raman state become equalized. The oscillation has a very small amplitude of $0.4 \%$, such that it is not visible in Fig. 2(c). With higher intensities of the control fields these oscillations decay and a CARS intensity suppression of more than $99.95 \%$ is achieved.

The limited suppression of the CARS intensity results from the fact that this scheme can only suppress a resonant contribution of a CARS intensity. However, due to the use of pulses with picosecond pulse duration the nonresonant contribution is lower than $0.2 \%(0.05 \%)$ for the case in which $|3\rangle(|4\rangle)$ acts as the control state. Nevertheless, the nonresonant contribution cannot be suppressed, and is anyhow influenced by the choice of the control light field pair, explaining the different amounts of the nonresonant contribution by a factor of 4 . The different amounts of nonresonant background occur as a population redistribution (which is slightly different for different control light field pairs) changes the optical properties of the molecule and thus also the $\chi^{3}$ which determines the nonresonant CARS intensity.

The molecular level scheme used here is a severe simplification of real molecules as additional vibrational, electronic, and Raman states are neglected. Those additional energy states might result in cascaded processes, in which population from the Raman state $|2\rangle$ or the electronic state $|3\rangle$ is transferred to upper states at higher energy. However, the application of the 
presented scheme would not be restricted by such cascaded processes as the simultaneous irradiation of the control light fields results in an optically induced coupling of the states $|1\rangle,|2\rangle$, and $|3\rangle$ resulting in an equal population in these states. If the population of one state is reduced due to a cascaded process introduced by the control light field pair, the changed population will automatically transfer to the two other states. As a result, the population in all three states would be reduced and an equal population of the three states would be found. Thus a suppression of CARS signal generation with the presented scheme would also be possible if cascaded processes additionally influence the population distribution of the molecule.

\section{TIMING CONSIDERATIONS}

For the applicability of this scheme it is crucial that at the time of CARS signal generation an equal population of the ground state and Raman state is present, while a coherence $\rho_{21}$ is not present. In order to maintain an equal population between the ground state and the Raman state for shorter population lifetimes of the Raman state $|2\rangle$ (e.g., down to 10 ps) the delay between the control pulses and the pump, Stokes, and probe pulses has to be reduced. Therefore, CARS signal generation is investigated for the case in which the control pulses and the pump, Stokes, and probe pulses are irradiated simultaneously.

Figure 3(a) depicts the CARS intensity as a function of the intensity of the control pulse pair for a delay of $\tau=0 \mathrm{ps}$ (black solid curve) and for comparison the already investigated case of $\tau=-30 \mathrm{ps}$ (blue dashed curve). It is found that due to the interaction of the probe pulses with a residual coherence $\rho_{21}$, generated by the control pulse pair, a CARS signal enhancement occurs with increasing control pulse intensity. However, with further increasing control intensities the CARS signal enhancement changes to a suppression of the CARS intensity as already known for a delay of $\tau=-30 \mathrm{ps}$. As CARS signal suppression can be achieved when all light fields are irradiated simultaneously, the presented scheme is applicable to CARS samples with rather short population lifetimes.

To understand the change from CARS signal enhancement to CARS signal suppression in Fig. 3(a), a closer look has to be taken at the coherence $\rho_{21}$ generated by the control pulse pair. As the lifetime of the coherence $\rho_{21}$ is shorter than the pulse duration of the control pulses the coherence already decays during the irradiation of the control pulses while, simultaneously, a decayed coherence is continuously regenerated by the control pulses. However, the regeneration of the coherence decreases during the irradiation of the control pulses, as the population difference between the involved energy states decreases, which inhibits the generation of a coherence between states. Note that this effect occurs because the population lifetime is longer than the coherence lifetime, which is the general case for CARS samples. As soon as all states are equally populated the generation of a coherence $\rho_{21}$ ceases completely. For high control pulse intensities equal population is already achieved by the leading edges of the control pulses, which contain only a fraction of the control pulse energy. With increasing intensity of the control pulses a smaller fraction of the pulses is required for an equalization

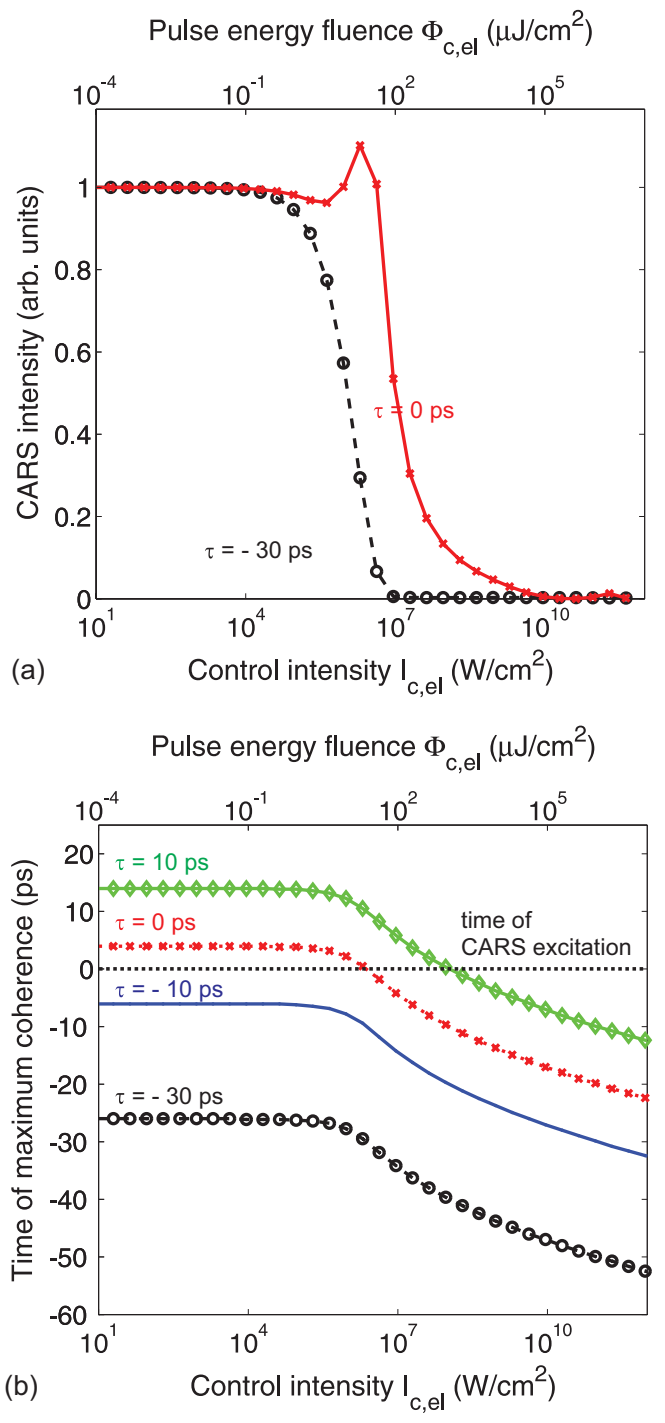

FIG. 3. (Color online) (a) CARS intensity as a function of the intensity of the control pulses for a delay of the control pulses of $\tau=0 \mathrm{ps}$ and $\tau=-30 \mathrm{ps}$. (b) Time of the maximum coherence $\rho_{21}$ generated by the control pulses as a function of the intensity of the control pulses for different delays $\tau$.

of all states. Consequently, the time at which the maximum coherence is generated by the control pulses depends on the intensity of the control pulses. Figure 3(b) depicts the time of the maximum coherence generated by the control pulses as a function of the intensity of the control pulses. Different delays between the control pulses and the pulses for CARS excitation are investigated. It can be seen that with increasing intensity of the control pulses the maximum coherence $\rho_{21}$ is generated earlier. An enhancement of CARS signal generation only occurs when the maximum coherence generated by the control pulses appears simultaneously with the irradiation of the probe pulse. For high intensities of the control pulses this is no longer the case but the maximum coherence is generated prior to the irradiation of the probe pulse and has already decayed until the time of CARS signal generation. On the contrary, the population of the states decays much slower and any imbalance between the population of the 


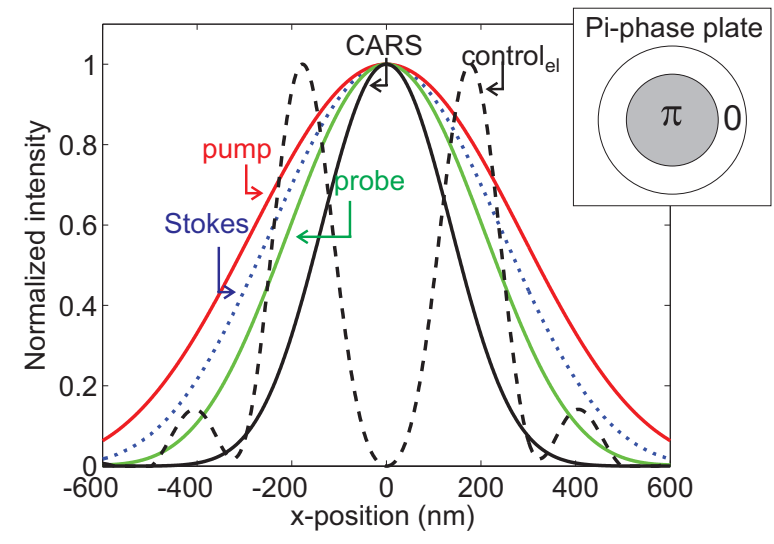

FIG. 4. (Color online) Cross sections of the spatial beam profiles of the pump (red solid curve), Stokes (blue dotted curve), probe (green solid curve), control (black dashed curve), and diffraction-limited CARS (solid black curve) light fields in the focal plane. The inset shows the phase plate used to create the donut-shaped beam profile of the control light fields.

states is automatically compensated due to the control pulses, i.e., an equal population of the involved states resembles an equilibrium condition during the irradiation of the sample with the control pulses. Consequently, the ground state and Raman state are equally populated at the time of CARS signal generation while no coherence $\rho_{21}$ is present, such that a saturable suppression of the CARS intensity is found.

\section{A. Spatial resolution improvement}

In order to estimate the achievable spatial resolution improvement of the proposed scheme, we simulated CARS images using control light fields with a donut-shaped spatial intensity modulation of the beam cross section. The control light field pair is assumed to use the electronic state $|3\rangle$ as the control state and is irradiated 30 ps prior to the pump, Stokes, and probe light fields. To create the donut-shaped intensity profiles of the control field pair, a phase mask (inset in Fig. 4, similar to the one used in [35]) is assumed to be in the beam path with an inner circle introducing a phase shift of $\pi$ with respect to the beam exterior, creating the same donut-shaped intensity profile in the focus for both control beams. The intensity profiles of the CARS excitation fields (i.e., pump, Stokes, and probe light fields) are modelled as unmodified Gaussian beams. The spatial profiles of the different light fields and the resulting CARS excitation profiles in the focus plane are then calculated assuming a microscope objective with numerical aperture 1.00. Cross sections of the beam profiles in the focal plane are depicted in Fig. 4.

Figure 5(a) shows cross sections of CARS excitation profiles for different total control intensities at the peak of the donut-shaped spatial profile $\left(I_{c}=0,2 \times 10^{6}, 2 \times 10^{7}, 2 \times 10^{8}\right.$, $\left.4 \times 10^{8} \mathrm{~W} / \mathrm{cm}^{2}\right)$. Increasing the control light field intensity results in a monotonous narrowing of the excitation profile significantly below the diffraction limit. As the diffractionlimited CARS excitation profile still has a relative intensity of $4.7 \%$ at the positions of the first minimum of the donut-shaped control light fields the CARS excitation profiles show small satellite peaks at these positions (Fig. 5, black dotted curve). These satellite peaks vanish when the donut peak intensity is increased sufficiently, such that also the intensity in this minimum leads to a strong suppression of the CARS intensity. Alternatively, the satellite peaks can be avoided by choosing a smaller numerical aperture (e.g., by a smaller beam diameter in front of the microscope objective), which results in a wider donut.

Two-dimensional illustrations of the diffraction-limited excitation profile of $318 \mathrm{~nm}$ width (FWHM) [dashed black curve in Fig. 5(a)] and a strongly narrowed excitation profile with a FWHM of $17.6 \mathrm{~nm}\left[I_{c}=4 \times 10^{8} \mathrm{~W} / \mathrm{cm}^{2}\right.$, dashed red curve in Fig. 5(a)] are given in Figs. 5(b) and 5(c), respectively. For a quantitative comparison, the FWHM of
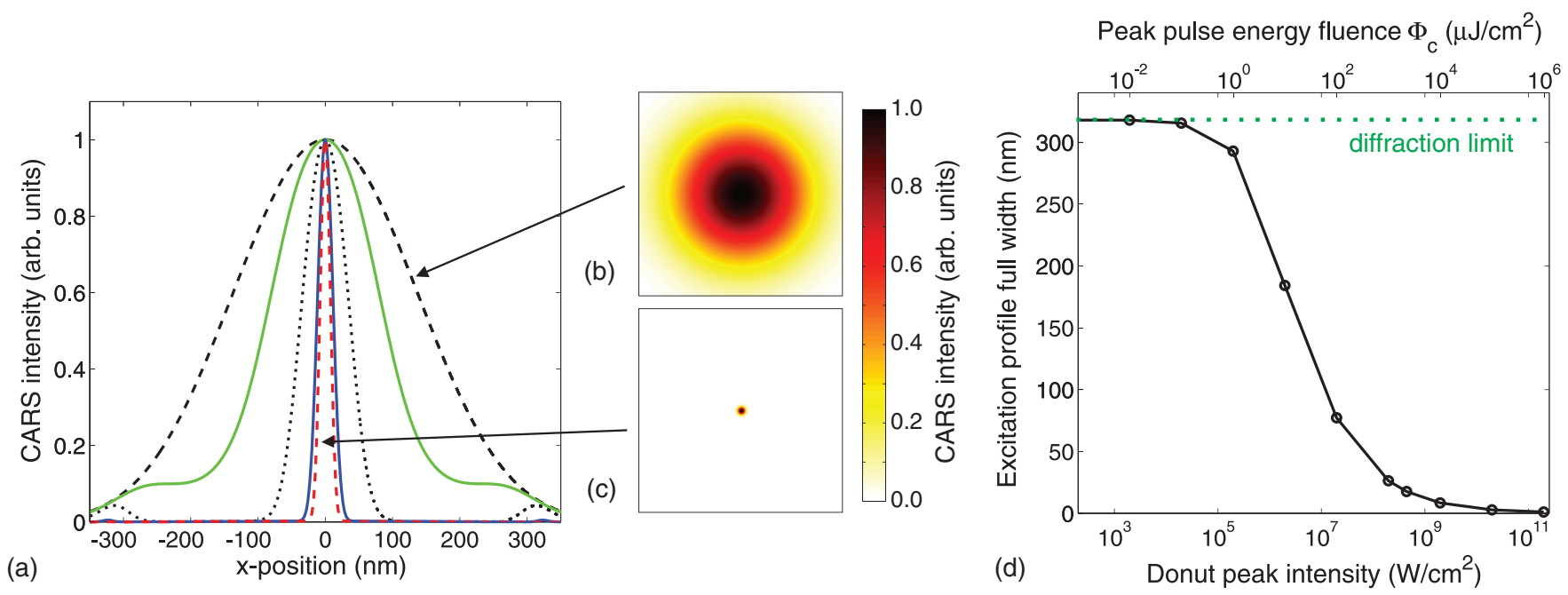

FIG. 5. (Color online) A donut-shaped spatial profile of the control light field pair narrows the CARS excitation profile below the diffraction limit. For different peak intensities of the donut the resulting CARS excitation profiles have been calculated. (a) Cross section of excitation profiles for donut peak intensities of $I_{c}=0,2 \times 10^{6}, 2 \times 10^{7}, 2 \times 10^{8}, 4 \times 10^{8} \mathrm{~W} / \mathrm{cm}^{2}$. Two-dimensional illustrations are shown for (b) $I_{c}=0 \mathrm{~W} / \mathrm{cm}^{2}$ [black dashed curve in (a)] and (c) $I_{c}=4 \times 10^{8} \mathrm{~W} / \mathrm{cm}^{2}$ [red dashed curve in (a)]. (d) FWHM of the excitation profiles as a function of the donut peak intensity. 


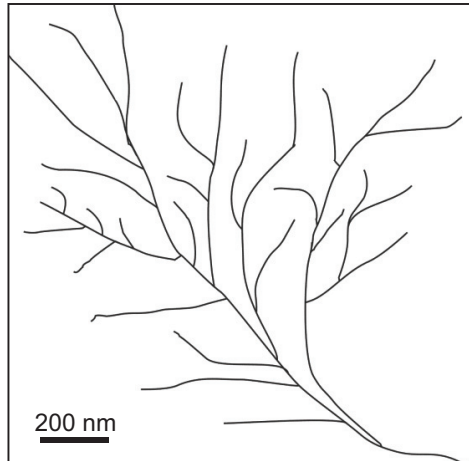

(a)

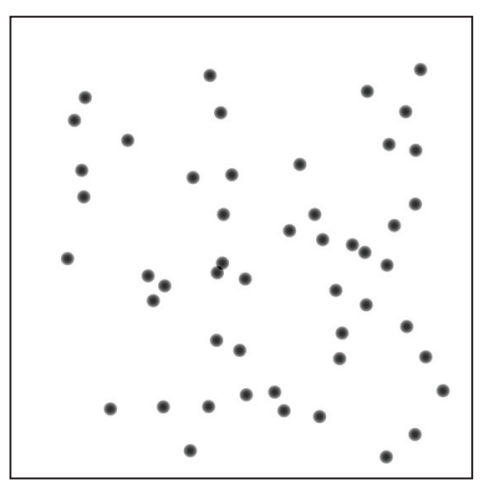

(d)

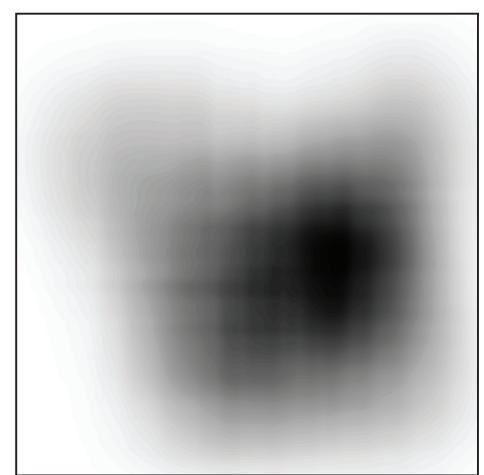

(e)

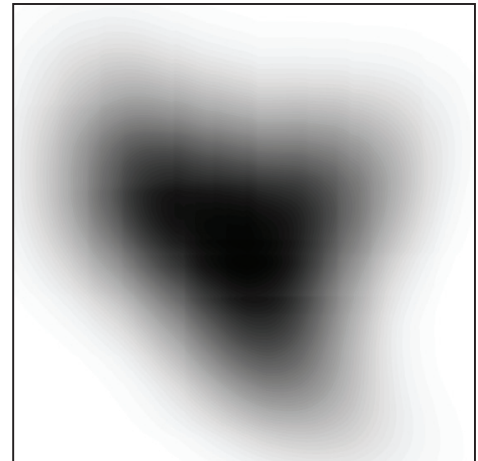

(b)

(c)
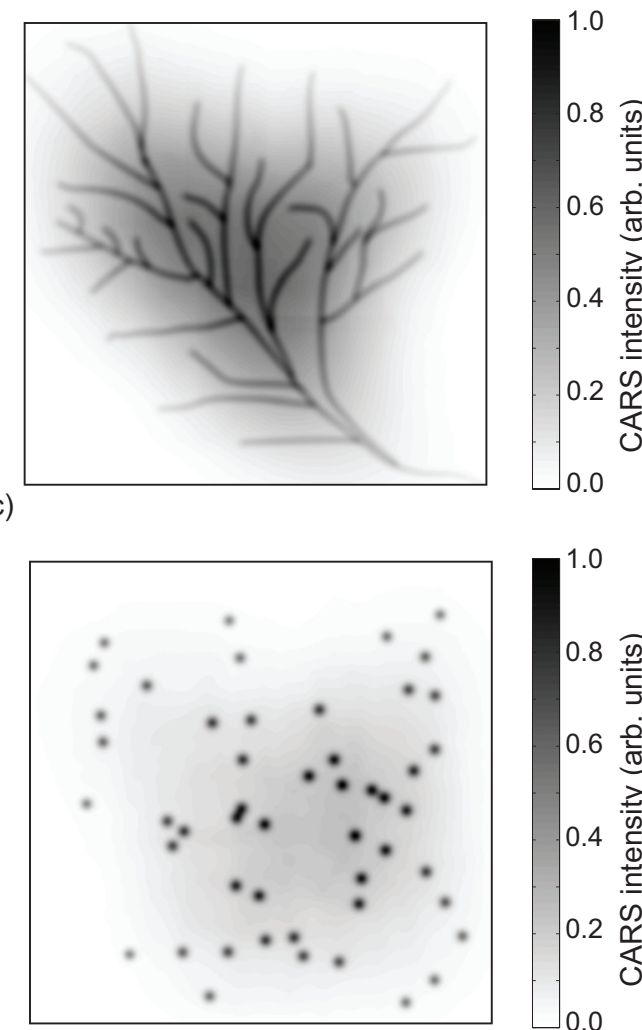

(f)

FIG. 6. Two test images have been created to simulate CARS images. (a) A dendritic line structure depicting, e.g., brain tissue is shown and (d) an ensemble of randomly located nanobeads (each $40 \mathrm{~nm}$ in diameter). (b) and (e) When no control light fields are applied a calculated CARS image does not contain any structure of the test images. (c) and (f) If the control light fields are applied the excitation profile is narrowed down to a FWHM of $17.6 \mathrm{~nm}$ and the CARS images reveal the original test images.

cross sections (which determines the spatial resolution for CARS microscopy) for control light field intensities spanning over a wide range are calculated and the results are displayed in Fig. 5(d) as a function of the peak donut intensity of the control light field. The CARS excitation width narrows monotonously from $318 \mathrm{~nm}$ (diffraction-limited) down to $1 \mathrm{~nm}$ at the highest intensity values, corresponding to a resolution enhancement by a factor of more than 300 .

In order to demonstrate that these excitation profiles result in CARS microscope images with sub-diffraction-limited resolution, we created two test images each of $1.34 \mu \mathrm{m} \times$ $1.34 \mu \mathrm{m}$ [Figs. 6(a) and 6(d)] and calculated the convolution [Figs. 6(b) and 6(e)] of the test images with a diffractionlimited excitation profile $(\mathrm{FWHM}=318 \mathrm{~nm})$, and the convolution [Figs. 6(c) and 6(f)] with an excitation profile narrowed below the diffraction limit [Fig. 5(c), FWHM = $\left.17.6 \mathrm{~nm}, I_{c}=4 \times 10^{8} \mathrm{~W} / \mathrm{cm}^{2}\right]$. The first test image resembles a dendritic line structure with varying spatial distances of less than $200 \mathrm{~nm}$, imitating, e.g., structures in brain tissue. The second test image consists of 50 randomly distributed nanobeads each of $40 \mathrm{~nm}$ diameter. In order to take into account the coherent nature of the CARS process the test images are convoluted with the excitation profile of the electric fields rather than the intensity distributions of the beam profile, and then the intensity image is calculated from the emerging CARS field in a second step.
In the first case of CARS imaging without control fields, the convolution of the dendritic structure with the diffractionlimited excitation profile leads to a blurred image with no visible features [Fig. 6(b)]. The convolution of the second test image with the same excitation profile also leads to a blurred image containing only information about the average density of the beads [Fig. 6(e)]. In contrast, if the excitation profile is significantly narrower than the diffraction limit the calculated CARS images clearly reveal information of the test images on a scale far below the diffraction limit [Figs. 6(c) and 6(f)]. The nonsuppressed nonresonant background of $0.2 \%$ results in a diffraction-limited background, however, the sub-diffraction-limited features are prominently displayed, such that a further image processing or additional schemes to remove the background are not required here.

The nonresonant signal found in these simulations is rather small as only four energy levels are used. In CARS experiments, especially when femtosecond pulses are used, the amount of a nonresonant contribution can be significantly higher. This results in an increase of the magnitude of the diffraction-limited background in Figs. 6(c) and 6(f) and consequently in a reduction of the contrast for structures on a scale below the diffraction limit. Also a nonresonant CARS signal of the surrounding of the resonant structure (for example, of an aqueous solution) would increase the magnitude of the diffraction-limited background. In the case in which 
the contrast for structures on a scale below the diffraction limit is too low due to the diffraction-limited background, additional schemes can be applied to remove it. Several known techniques can be applied to reduce nonresonant contributions of the CARS signal, such as heterodyne detection [33], spectral focusing [36], or time-delayed CARS [37]. It is also possible to calculate a difference image between two CARS images, one with and one without an applied control light field pair. It has been shown that this can provide sub-diffraction-limited spatial resolution for a case corresponding to a magnitude of the nonresonant intensity of $25 \%$ of the resonant CARS intensity [19]. Consequently, the presented scheme can also be used to generate CARS images with sub-diffraction-limited resolution when a high nonresonant signal is generated by the sample and the surrounding area, e.g., by an aqueous solution.

An experimental realization of this scheme requires a complex setup and sophisticated optical elements. However, this is no principle limitation as optical elements to combine different beams of very different wavelengths (UV, VIS, IR) are readily available. Suitable laser sources would be optical parametric oscillators or optical parametric amplifiers. These are commercially available, delivering pulses from the UV to the IR with pulse energies of more than several $\mu \mathrm{J}$. Thus there should be no technical limitations to experimentally evaluate the proposed scheme for sub-diffraction-limited spatial resolution in CARS microscopy.

\section{CONCLUSION}

In summary we presented a numerical investigation of a scheme providing sub-diffraction-limited CARS microscopy based on stimulated emission pumping. The equalization of the population in the ground state and Raman state provides the capability to suppress the CARS intensity to a large extent, in our example by more than $99.8 \%$. The required intensities and pulse energy fluences were estimated using an exemplary sample based on quantum dots [31]. Using adequate spatial profiles for the different light fields, we calculated expected CARS excitation profiles under the influence of the control light field pair. Thereby we found a narrowing of the excitation profile widths significantly below the diffraction limit, corresponding to a theoretical resolution enhancement of up to 300 for estimated intensity values of up to $100 \mathrm{GW} / \mathrm{cm}^{2}$.

Sub-diffraction-limited spatial resolution was numerically demonstrated using two computer-generated test images. For both test images, the calculated CARS image revealed subdiffraction-limited features with a resolution of approximately $17.6 \mathrm{~nm}$. In this calculation a total intensity of the control light field pair of $400 \mathrm{MW} / \mathrm{cm}^{2}$ and a pulse energy fluence of $4 \mathrm{~mJ} / \mathrm{cm}^{2}$ was used. The intensity values used are comparable to those used in STED microscopy and a comparable spatial resolution was found [38]. Using a laser system with a typical repetition rate of $80 \mathrm{MHz}$ the intensity value used corresponds to an average power of approximately $10 \mathrm{~mW}$.

As the approach is solely based on the optical stimulation of transitions, only general molecular properties had to be assumed, i.e., no sample specific properties were exploited. It was demonstrated and explained that the population and coherence lifetimes can span a wide range of values without restricting the applicability of the presented scheme. Consequently, this approach is, in principle, applicable to any CARS sample and together with the requirements to the intensities of the control pulse pair the calculations resemble a case that should be experimentally feasible.
[1] R. Hellwarth and P. Christensen, Opt. Commun. 12, 318 (1974).

[2] M. Müller, J. Squier, K. R. Wilson, and G. J. Brakenhoff, J. Microsc. 191, 266 (1998).

[3] A. Zumbusch, G. R. Holtom, and X. S. Xie, Phys. Rev. Lett. 82, 4142 (1999).

[4] J.-X. Cheng and X. S. Xie, J. Phys. Chem. B 108, 827 (2004).

[5] X. Nan, J.-X. Cheng, and X. S. Xie, J. Lipid Res. 44, 2202 (2003).

[6] M. Jurna, M. Windbergs, C. J. Strachan, L. Hartsuiker, C. Otto, P. Kleinebudde, J. L. Herek, and H. L. Offerhaus, J. Innov. Opt. Health Sci. 2, 37 (2009).

[7] Y. Wang, C.-Y. Lin, A. Nikolaenko, V. Raghunathan, and E. O. Potma, Adv. Opt. Photon. 3, 1 (2011).

[8] S. W. Hell and J. Wichmann, Opt. Lett. 19, 780 (1994).

[9] E. Betzig, G. H. Patterson, R. Sougrat, O. W. Lindwasser, S. Olenych, J. S. Bonifacino, M. W. Davidson, J. LippincottSchwartz, and H. F. Hess, Science 313, 1642 (2006).

[10] B. Huang, S. A. Jones, B. Brandenburg, and X. Zhuang, Nat. Methods 5, 1047 (2008).

[11] V. Westphal, S. O. Rizzoli, M. A. Lauterbach, D. Kamin, R. Jahn, and S. W. Hell, Science 320, 246 (2008).

[12] Dietmar W. Siemann and Peter C. Keng, Cancer Research 46, 3556 (1986).
[13] V. Raghunathan and E. O. Potma, J. Opt. Soc. Am. A 27, 2365 (2010).

[14] H. Kim, G. W. Bryant, and S. J. Stranick, Opt. Express 20, 6042 (2012).

[15] K. M. Hajek, B. Littleton, D. Turk, T. J. McIntyre, and H. Rubinsztein-Dunlop, Opt. Express 18, 19264 (2010).

[16] W. P. Beeker, P. Groß, C. J. Lee, C. Cleff, H. L. Offerhaus, C. Fallnich, J. L. Herek, and K.-J. Boller, Opt. Express 17, 22632 (2009).

[17] W. P. Beeker, C. J. Lee, K.-J. Boller, P. Groß, C. Cleff, C. Fallnich, H. L. Offerhaus, and J. L. Herek, Phys. Rev. A 81, 012507 (2010).

[18] C. Heinrich, S. Bernet, and M. Ritsch-Marte, Appl. Phys. Lett. 84, 816 (2004).

[19] C. Cleff, P. Groß, C. Fallnich, H. L. Offerhaus, J. L. Herek, K. Kruse, W. P. Beeker, C. J. Lee, and K.-J. Boller, Phys. Rev. A 86, 023825 (2012).

[20] C. H. Hamilton, J. L. Kinsey, and R. W. Field, Annu. Rev. Phys. Chem. 37, 493 (1986).

[21] B.-N. Dai and P. Lambropoulos, Phys. Rev. A 36, 5205 (1987).

[22] B. Schrader and W. Meier, Raman/IR Atlas (Verlag Chemie, Weinheim, 1974).

[23] P. W. Milonni and J. H. Eberly, Lasers (Wiley, New York, 1988). 
[24] E. J. Heilweil, R. R. Cavanagh, and J. C. Stephenson, Chem. Phys. Lett. 134, 181 (1987).

[25] T. A. Cellucci and E. R. Nixon, J. Phys. Chem. 89, 1991 (1985).

[26] M. R. Lewis, T. Marshall, E. H. Carnevale, and F. S. Zimnoch, Phys. Rev. 164, 94 (1967).

[27] M. Hofmann and H. Graener, Chem. Phys. 206, 129 (1996).

[28] Zhaohui Wang, Andrei Pakoulev, and Dana D. Dlott, Science 296, 2201 (2002).

[29] A. J. Wurzer, T. Wilhelm, J. Piel, and E. Riedle, Chem. Phys. Lett. 299, 296 (1999).

[30] F. Vallée and F. Bogani, Phys. Rev. B 43, 12049 (1991).

[31] H. Kamada, H. Gotoh, J. Temmyo, T. Takagahara, and H. Ando, Phys. Rev. Lett. 87, 246401 (2001).
[32] C. Ventalon, J. M. Fraser, M. H. Vos, A. Alexandrou, J.-L. Martin, and M. Joffre, Proc. Natl. Acad. Sci. USA 101, 13216 (2004).

[33] E. O. Potma, C. L. Evans, and X. S. Xie, Opt. Lett. 31, 241 (2006).

[34] John C. Deàk, Lawrence K. Iwaki, Stuart T. Rhea, and Dana D. Dlott, J. Raman Spectrosc. 31, 263 (2000).

[35] T. A. Klar, S. Jakobs, M. Dyba, A. Egner, and S. W. Hell, Proc. Natl. Acad. Sci. USA 97, 8206 (2000).

[36] T. Hellerer, A. M. K. Enejder, and A. Zumbusch, Appl. Phys. Lett. 85, 25 (2004).

[37] A. Volkmer, L. Book, and X. Xie, Appl. Phys. Lett. 80, 1505 (2002).

[38] Volker Westphal and Stefan W. Hell, Phys. Rev. Lett. 94, 143903 (2005). 